\title{
An in-flight investigation of a turbulent boundary layer at Reynolds numbers up to $\operatorname{Re}_{\theta}=49,400$
}

\author{
Christina Dunker ${ }^{1}$ (D)
}

Received: 26 April 2020 / Revised: 6 September 2020 / Accepted: 6 October 2020 / Published online: 23 December 2020

(C) The Author(s) 2020

\begin{abstract}
The ongoing attempts to gain access to the realm of high Reynolds number turbulence have resulted in the dedicated development of major experimental facilities and novel diagnostic methodologies as well as in the probing of atmospheric surface flows. In contrast to this, the presented study discusses the feasibility of an in-flight laboratory for Reynolds number investigations up to $\operatorname{Re}_{\theta} \leq 49,400$. The underpinning velocity data were obtained in flight tests by two moveable differential pressure probes and a stereo Particle Image Velocity (sPIV) system. The region of interest was located far downstream of the aircraft's nose within the fuselage boundary layer. The pressure probes scanned the full boundary layer while the sPIV system remained fixed at certain wall-normal locations. The velocity data acquired exhibits distinct characteristics within the defect layer that deviate from Coles' classical description of the wake. Furthermore, the streamwise turbulence intensities show a pronounced 'outer peak' further away from the wall at $y^{+}=2000-5000$. The measurements were conducted under authentic flight conditions with an increased level of free-stream turbulence. These boundary conditions enabled an analysis of turbulent flows that are of relevance for various aeronautical applications. The manuscript elaborates on the main findings of this experimental study by presenting the velocity profiles captured by the moveable pressure probe system and samples of sPIV data. The capabilities and limitations of a flying laboratory for the investigation of high Reynolds number turbulence are discussed in detail.
\end{abstract}

\section{Graphic Abstract}

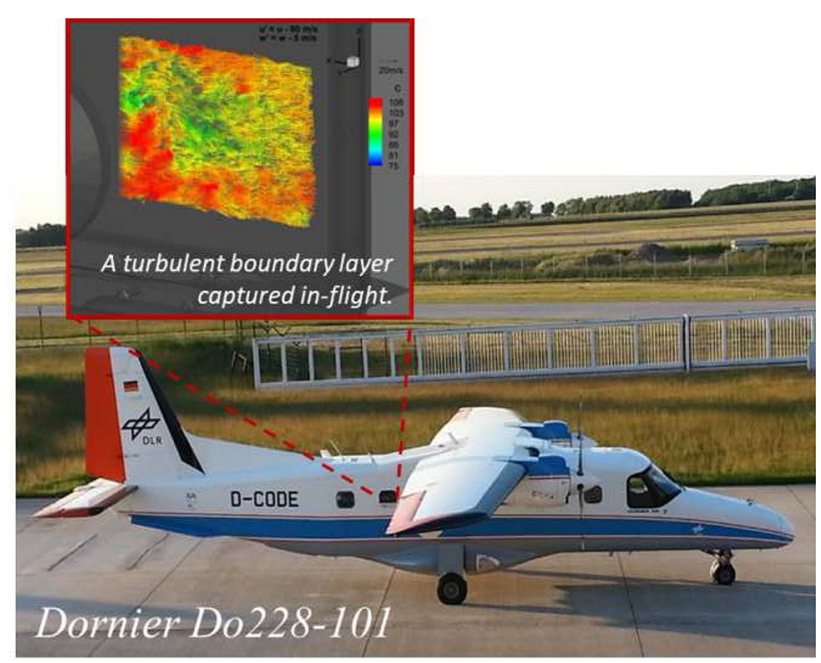

Christina Dunker

c.dunker@ rocketlab.co.nz

1 Department of Experimental Methods, German Aerospace

Center, Bunsenstr. 10, 37073 Göttingen, Germany 


\section{Introduction}

Various technologically and geophysically driven flows are at comparably high Reynolds numbers and demand a thorough elucidation of the underlying physical laws. Furthermore, current turbulent boundary layer models strongly rely on a firm description of turbulent flows. Experimental and numerical simulations are striving to close the gaps while exposing limitations of the theories and methodologies, such as spatial resolution effects of hot-wires (Hutchins et al. 2009) or the lagging computational resources for fully resolved direct numerical simulations with increasing Reynolds numbers.

The replication of ideal conditions in laboratory environments is a complex task that comes at high expenses to construct and operate facilities for high Reynolds number experiments. Nevertheless, new facilities such as the $111.5 \mathrm{~m}$ long tube of the Centre for International Cooperation in Long Pipe Experiments (CICLoPE) (Örlü et al. 2017) that operates at ambient pressures became available recently. This configuration achieves Reynolds numbers of up to $\operatorname{Re}_{\tau}=40,000$ while keeping the viscous scales within a moderate range that alleviate the accessibility for measurement techniques. The friction Reynolds number is defined as $\operatorname{Re}_{\tau}=\delta_{99} u_{\tau} / v$ where $\delta_{99}$ is the boundary layer thickness at $99 \%$ of the free-stream velocity, $u_{\tau}$ is the friction velocity and $v$ is the kinematic viscosity. The Princeton Superpipe (Marusic et al. 2010; Zagarola and Smits 1998) is capable of generating extremely high Reynolds numbers of $\operatorname{Re}_{\tau}=100,000$ when operating at pressures well above atmospheric to lower the kinematic viscosity. This, however, results in a significant decrease of the smallest turbulent scales and of the spatial resolution of any probe.

In contrast to that, the near-neutral atmospheric surface layer (ASL) offers unprecedented conditions for the exploration of turbulent flows at high Reynolds numbers up to $\operatorname{Re}_{\tau} \sim O\left(10^{6}\right)$. Andreas et al. (2006) conducted experiments over the horizontally homogeneous and unobstructed Antarctic sea ice on Ice Station Wedell (ISW). The findings of the study contributed to discussions surrounding the universality of the von Kármán constant. The authors hypothesized that the von Kármán constant shows no correlation with roughness Reynolds numbers and hence claimed a unification of laboratory (Österlund et al. 2000) and atmospheric observations regarding the definition of the von Kármán constant. Various field campaigns were conducted at the Surface Layer Turbulence and Environmental Science Test (SLTEST) facility on the salt flats of the Great Salt Lake Desert (Metzger et al. 2007). Hutchins et al. (2012) carried out several experiments in this environment with large sensor arrays to investigate turbulent structures. The mean statistics and two-point correlation maps indicated that the near-neutral ASL comes very close to the logarithmic region of canonical flat plate turbulent boundary layer flow and that many findings agree with laboratory studies. Super-structures of kilometres scale that are spanning over more than $10 \delta$ in length were observed. These enormous turbulent structures point to an extremely wide spectrum of different scales of vortical dynamics in the atmosphere. A similar test facility compared to the SLTEST site was established on a flat bed of the Qingtu Lake in China (Wang and Zheng 2016; Lui et al. 2017). The Quingtu Lake Observation Array (QLOA) is a three-dimensional array with numerous towers that were equipped with thermometers and sonic anemometers. Experiments in this environment were able to resolve long meandering structures and their inclination angles by means of two-point correlation of the fluctuating streamwise velocity as well as spectral analysis under different thermal stability conditions.

The utilisation of optical-based methods such as Particle Image Velocimetry or smoke visualization allowed a more comprehensive spatial characterisation of coherent structures in the higher Reynolds number regime. Hommema and Adrian (2003) identified hairpin vortex packets in very high Reynolds number flows at the aforementioned SLTEST facility. The high spatial resolution of the experiments unravelled the range of spatial dimensions of hairpin vortex packets. A super-large-scale Particle Image Velocimetry (SLPIV) approach with natural snowfall as flow tracers was introduced by Toloui et al. (2014) and Heisel et al. (2018). Optical based methods such as SLPIV can gain non-intrusive access to complex spatial structures such as forward-inclined vortex structures and could link findings of laboratory studies to observations in the ABL. In general, difficulties when probing the ASL arise from nonstationarity; limited spatial resolution of sensor arrays that are facing turbulent structures of enormous scales; the diurnal cycle that triggers unstable conditions; polluted wind (with sand grains or dust entrainments); as well as roughness effects.

Conceptually, an experiment set up with a full-scale aircraft or a marine vessel (Utama et al. 2018) could bridge the gap between the existing wind tunnel facilities and ASL experiments. Furthermore, various aeronautical applications could strongly benefit from the experimental approach that is presented in this paper. At the current state of development the design of a commercial aircraft still requires proof-of-concept based on tests which assure full dynamic similarity with respect to Mach and Reynolds numbers in the free atmosphere. The research project HINVA (High lift INflight VAlidation) (Rudnik and Schwetzler 2016; Dunker and Geisler 2018) for example combined numerical simulations; wind tunnel tests; as well as flight tests with a 
full-scale commercial aircraft to investigate the transferability of numerical and experimental simulations to in-flight performance estimations of high-lift devices. A multifaceted approach like this significantly improves the accuracy in predicting the maximum lift of an aircraft.

The present paper extends this discussion further by introducing an aircraft as an investigative environment for wall-bounded turbulence research. Two differential pressure probes were installed on a moveable stem outside of the aircraft's fuselage and a stereoscopic Particle Image Velocimetry (sPIV) installation captured all three velocity components close to the stem. Politz et al. (2013) are summarizing further findings of this experiment with regard to the sPIV measurement as well as technical aspects of this flight test installation and its certification process. Information on the lateral and normal velocity component in this flow regime are quit scarce since most of the experimental simulations so far have resorted to hot-wire or Pitot probes (Marusic et al. 2010). The focus of this paper is on the data analysis of this new experimental implementation since this is the novel element.

\section{Flight tests-setup}

The experimental equipment of this flight test campaign comprised two shiftable differential pressure probes; a stereo Particle Image Velocimetry (sPIV) system; an Interferometric Laser Imaging for Droplet Sizing (ILIDS) installation; and an on-board data acquisition unit that accessed the flight attitude database of the aircraft. All systems and devices were located inside the cabin of a commuter aircraft of type Dornier Do228-101 (see Fig. 1). This aircraft was allocated and operated by the Flight Operation Center of the German Aerospace Center (DLR) in Braunschweig/Germany. The fuselage of this type of aircraft is not circular, but rectangular in shape and hence simulates a flat plate surface. The region of interest within the flow was located at the position of the penultimate cabin window on the starboard side. This downstream placement ensured an extended run-up distance from the nose of the fuselage to the measurement window $1=8.4 \mathrm{~m}$ downstream. The propeller plane was located roughly $5.7 \mathrm{~m}$ upstream of the measurement window. The minimum distance between the blade tips of the propeller and the fuselage was $525 \mathrm{~mm}$. The specified Revolutions Per Minute (RPM) of the propeller in cruise flight were 1512 RPM according to the Pilot's Operating Handbook (Dornier Luftfahrt GmbH 1991). The impact of the propeller slipstream on the energy spectra is discussed in Sect. 5.1 and is depicted in Fig. 8.

The standard cabin window of the non-pressurised fuselage was replaced by a rigid aluminium frame with a high-quality optical glass pane; a break-through for the pressure probe stem; as well as a small hole for the static pressure tap (see Fig. 2). The differential pressure system with its two Pitot probes was integrated into the shiftable stem that continuously moved through the boundary layer

Fig. 1 Flight test installation (units: mm). 1: Measurement window; 2: Moveable stem; 3: Outer Pitot probe; 4 : Boundary layer probe; 5: Static pressure tapping; 6: sPIV camera; 7: Sketched laser light sheet
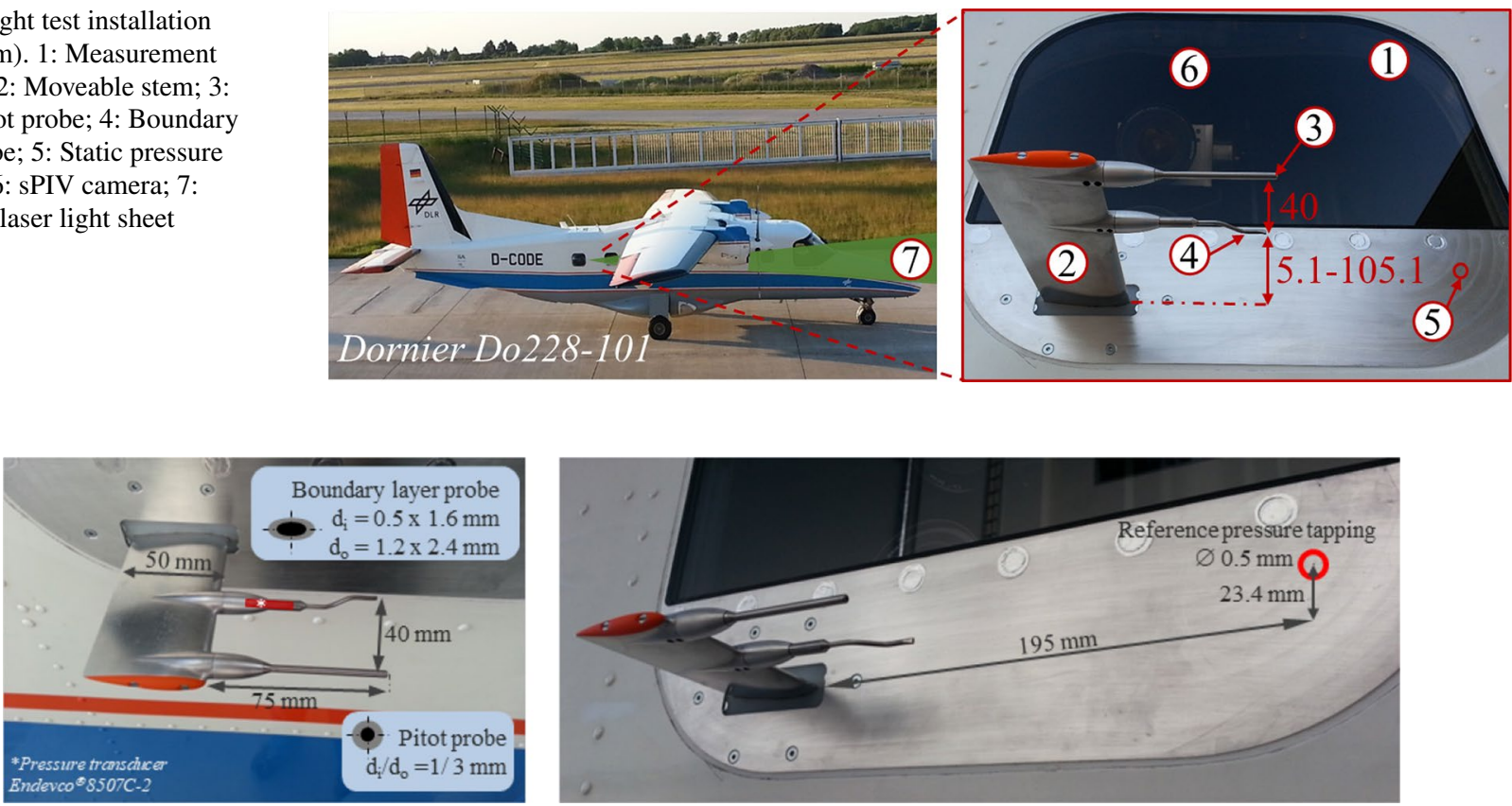

Fig. 2 Dimensions of the differential pressure system as integrated into the moveable stem and the aluminium frame of the measurement window 
in a wall-normal direction. The stem was a small rectangular wing with a chord length of $50 \mathrm{~mm}$ and a NACA0015 airfoil shape (see Fig. 2).

A straight Pitot probe with a diameter ratio of $\theta=0.33$ and a round tip was attached to the far end of the stem. The probe adapted a more robust design to make it less susceptible to yaw sensitivity and mechanical damage during flight or ground-handling. The second so-called boundary layer probe was positioned closer to the surface of the fuselage. Its purpose was to capture the streamwise velocity information from locations as close as possible to the wall. This led to a curved probe shape with a flattened opening and an outer diameter ratio of $\theta=0.67$. This design was intended to reduce deviations of the true total pressure caused by the specific finite size of the probe body in close proximity to the surface. Further details on the dimension of the probes are given in Figure 2. The minimum distance between the centre of the boundary layer probe and the outer fuselage wall was $y_{\text {blp,min }}=5.1 \mathrm{~mm}$. The maximum separation between the outer Pitot probe and the wall was $y_{\mathrm{pp}, \max }=145.1 \mathrm{~mm}$. The two probes were $40 \mathrm{~mm}$ apart. The distance between the leading edge of the stem and the tip of the probes was around $75 \mathrm{~mm}$.

The piezo-resistive pressure transducer of the boundary layer probe was of type Endevco ${ }^{\circledR} 8507 \mathrm{C}-2$. The sensor covered a pressure range of $0-2 \mathrm{psiG}$. This miniature transducer with a diameter of $2.3 \mathrm{~mm}$ was located inside the boundary layer probe $42 \mathrm{~mm}$ away from its opening to allow a fast frequency response. However, it was anticipated that the significant changes of the ambient temperatures between departure and ceiling altitude during the experiments would cause a drift in the sensor signals that required careful corrections. Therefore, a different strategy was implemented for the sensor of the Pitot probe. The pressure transducer of the outer Pitot probe was a Kulite ${ }^{\circledR}$ XCL072-0.35 barG that was placed inside the temperatureregulated cabin of the aircraft. A tube of $900 \mathrm{~mm}$ length connected the pressure transducer and the Pitot probe. The resulting gain in measurement certainty came with an increase in pressure attenuation. A post-experiment frequency analysis indicated a damped pressure signal of the Pitot probe sensor. The frequency response of the boundary layer probe with a sensor closer to the opening was also compromised. A significant damping of the pressure signal was observed above frequencies of $1000 \mathrm{~Hz}$ (see Fig. 8). This attenuation was caused by a Helmholtz resonance in the boundary layer probe that was induced by the cavity between the opening of the probe and the sensor itself. Frequencies beyond that threshold are damped and cannot be used for further analysis. Both differential pressure sensors were calibrated by applying static pressures to the pneumatic system. No dynamic calibration, which would resolve the frequency response of the system, was carried out. Hence, a Bode plot cannot be provided.

The reference pressure tapping for both differential pressure sensors was located $195 \mathrm{~mm}$ upstream and slightly displaced from the extension of the chord line of the stem to avoid interferences between the stem and the static pressure readings (see Fig. 2). A barometric pressure transducer of type Setra $278-600 \mathrm{M}$ with a pressure range of $610-1100 \mathrm{hPa}$ was connected to the static tap of diameter $d_{s}=0.5 \mathrm{~mm}$. This transducer was installed inside the cabin and it was connected to the tubing system that tapped the differential pressure reference tubes of all pressure sensors.

The sPIV/ILIDS light sheet was oriented parallel to the aircraft fuselage enabling two measurement planes at $\mathrm{y}_{P I V, \max }=72 \mathrm{~mm}$ and $\mathrm{y}_{P I V, \text { min }}=41.5 \mathrm{~mm}$ wall distance (Fig. 3). The light sheet thickness was $\mathrm{b}_{\text {SPIV }}=2 \mathrm{~mm}$. The light source was a dual-head Nd:YAG laser emitting monochromatic green light at $532 \mathrm{~nm}$ with an output power of around $170 \mathrm{~mJ}$ and a pulse length of $9 \mathrm{~ns}$. The light sheet was expanded and redirected with the help of three lenses and a mirror. Misalignment uncertainties due to small dislocations of the laser light sheet relatively to the focused plane of the camera were compensated by means of disparity corrections during the evaluation process. Two pco.1600 PIV cameras with a resolution of $1600 \mathrm{px} \times 1200 \mathrm{px}$ operated at a frame rate of $10 \mathrm{~Hz}$. These cameras were aligned under a stereoscopic angle of $45^{\circ}$ relative to each other in which the lower camera was looking horizontally at the light sheet plane. The resulting field of views of the two wall-parallel planes were $75 \times 100 \mathrm{~mm}^{2}$ (magnification factor $\mathrm{M}_{f}=16 \mathrm{px} / \mathrm{mm}$ ) and $63 \mathrm{px} \times 84 \mathrm{~mm}^{2}$ for the plane closer to the wall (magnification factor $\mathrm{M}_{f}=19 \mathrm{px} / \mathrm{mm}$ ). Hydrometeors such as cloud or haze droplets served as seeding sources for sPIV. This necessitated measurements in environments that have higher ambient turbulence levels and hence partially impeded the acquisition of undisturbed velocity profiles.

The purpose of ILIDS was solely the generation of complementary data for the performance assessment of the SPIV system that relied on polydispersed hydrometeors in the atmosphere as seeding source. The ILIDS system utilized a GX3300 camera with a resolution of $3296 \mathrm{px} \times 2472 \mathrm{px}$ in combination with a Makro-Planar $100 \mathrm{~mm}$ lens and a slit aperture. The field of view of this non-intrusive droplet sizing sensor was slightly upstream of the sPIV installation and covered an area within the light sheet of $21.5 \times 28.7 \mathrm{~mm}^{2}$. ILIDS is capable of measuring droplet size distributions based on the evaluation of out-of-focus images that contain the light scattering signatures of droplets (so called interferograms). This method essentially unravels the physical and optical characteristics of the captured interferograms by means of Mie's theory. The system dynamic varies within the range of $10 \leq d_{\mathrm{p}, \max } / d_{\mathrm{p}, \min } \leq 41$. This particular ILIDS 


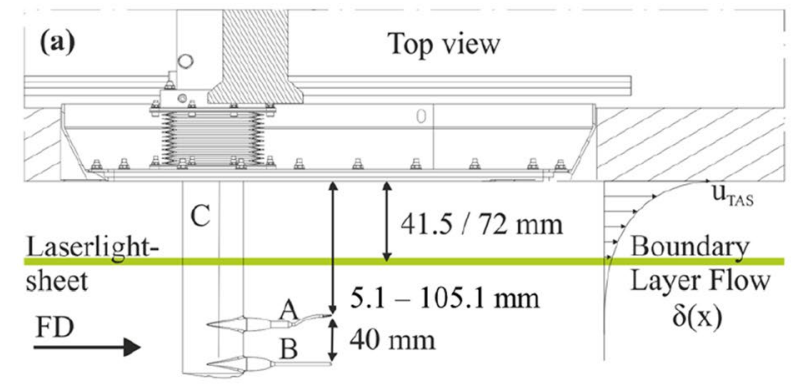

A: Boundary Layer Probe B: Pitot Probe

C: Traversing Probe Stem
FOV: Field of View

FD: Flight Direction

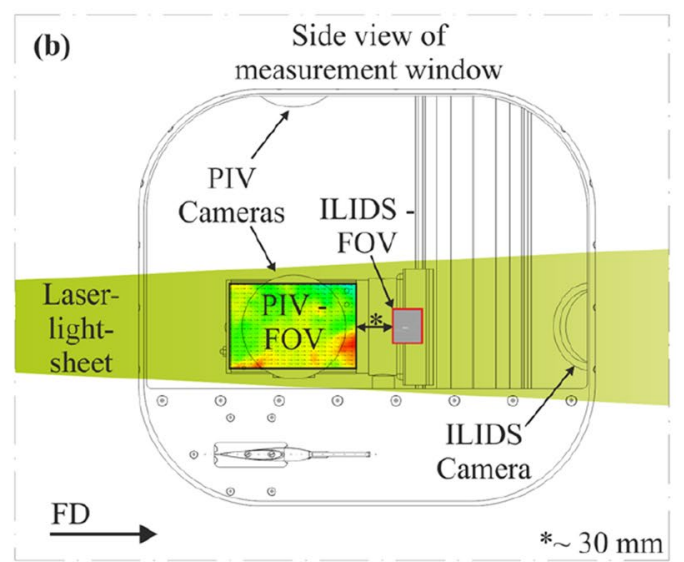

Fig. 3 Top view (a) and side view (b) of the measurement window. The pressure probe stem was continuously moving through the boundary layer whereas the laser light sheet and hence the sPIV/ILIDS measurement plane was at a fixed position during each flight

setup resolved hydrometeors as small as $d_{\mathrm{p}, \min }=5.8 \mu \mathrm{m}$ and as large as $d_{\mathrm{p}, \max }=236 \mu \mathrm{m}$. Laboratory experiments with monodispersed PMMA particles indicated that the accuracy of this method strongly depends on the optical setup as well as the ILIDS evaluation method. Dunker et al. (2016) discussed the potential of ILIDS for cloud droplet characterisation, the accuracy of the methodology itself as well as results of this flight test campaign.

\section{Measurement procedures}

In total, with more than $13 \mathrm{~h}$ of flight time and over $3550 \mathrm{~km}$ covered, several thousand sPIV and ILIDS images were acquired. Each of the flights comprised between six to nine measuring cycles of roughly 6 min length. The recording procedure of each measuring cycle was split into two parts. After entering a suitable cloud layer and ensuring the safe operation of the laser, all three measurement systems were activated simultaneously. During the first three minutes of recording time, the sPIV/ILIDS and the pressure probe units were capturing data in parallel. The pressure probes were positioned at a fixed distance away from the wall very close to the sPIV plane. After three minutes recording time at steady flight conditions the sPIV/ILIDS system stopped acquiring data and the pressure probe stem now moved independently through the whole boundary layer. The step motor of the stem was programmed to reposition the probes every three seconds. Each profile consisted of 37 to 49 different positions within the boundary layer. The turnover time $\mathrm{t}_{L}=\delta_{99} / \mathrm{u}_{\infty}$ of the presumable largest eddy is estimated using a measuring cycle that exhibited the thickest boundary layer of the herewith presented data base (see C150 in Table 2). The resulting time scale is roughly 0.0015 seconds.
The number of captured large eddy turnovers within the three seconds interval is 2043 which is adequate for the statistical validation of velocity information.

The parameter space covered different indicated airspeeds ranging from $120,130,140$ to $180 \mathrm{kt}$ in combination with retracted flaps or flaps set to an angle of $20^{\circ}$. All flights were conducted during night time to avoid the negative influence of sunlight on the sPIV/ILIDS images and to simplify the flight test procedures with respect to laser safety. More information on the procedures for a safe in-flight laser operation are provided by Politz et al. (2013).

The combination of wall-normal scans with the high temporal resolution provided by the pressure sensors and the wall-parallel, highly-spatially resolving sPIV plane allowed a thorough analysis of the viscous flow, covering the outer layer including the defect layer.

\section{Data analysis and accuracy}

\subsection{Data preselection}

The free-flight conditions of this experiment posed several challenges for the boundary layer analysis. The high level of ambient turbulences when flying through clouds resulted in varying flight speeds and increased turbulence intensities during the data acquisition intervals of each flight. The initial phase of data processing was hence dedicated to the definition of selection thresholds (e.g. acceptable variations of the flight speed: $\pm 1 \mathrm{~m} / \mathrm{s}$ ) and identification of adequate velocity profiles. Points measured at slow flying speeds with flaps extended, often exhibited strong velocity variations of up to $\pm 5 \mathrm{~m} / \mathrm{s}$ combined with a phugoid motion of the aircraft. Profiles of high quality were almost always recorded 
when flying at highest speeds around $u_{\text {TAS }}=100-110 \mathrm{~m} / \mathrm{s}$ due to the diminishing impact of gusts and phase-shifted piloting inputs that changed the aircraft attitude.

\subsection{Correction of the differential pressure sensor system}

The calibration and correction of the differential pressure sensor system comprised the adjustment of the pressure probe displacement due to the velocity gradient close to the wall (Princeton displacement correction McKeon et al. 2003) as well as the compensation of the thermal drift of the offset voltage. The latter measure relied on total pressure information that was allocated by the aircraft's Pitotstatic system. This total pressure in combination with the local static pressure measured by the wall tapping was used to correct the impact pressure at the boundary layer edge of each acquired velocity profile (Gyorgyfalvy 1965). Corrections that compensate for Reynolds number effects, turbulence, directional sensitivity, and wall proximity (Bailey et al. 2013) were found to be ineffectual. The static pressure measured by the local wall tapping was lowered by $0.6 \cdot \tau_{w}$ to correct the effects of finite depth, hole size and burrs.

\subsection{Measurement resolution}

The finite size of the differential pressure probes and of the sPIV interrogation volumes are introducing attenuation effects in the spatial domain. Table 1 lists the experimental parameters expressed in viscous units. The dimensions of the smallest interrogation window size $48 \mathrm{px} \times 48 \mathrm{px}$ is quite similar to the resolution of both pressure probe units. In general, the spatial resolutions of the measurement systems are comparably poor and it is safe to assume that the energy contribution of smaller-scale eddies remained unresolved. This lack of spatial resolution results in an underestimation of variance statistics, which increases towards the wall where contributions of small, viscous-scaled eddies prevail.

The selection of the sPIV interrogation window size is subject to two competing effects. Whilst a small interrogation window will reduce the negative effects of spatial filtering, larger windows will increase the number of matched particle pairs and therefore the detection probability at low particle image densities. Figure 4 collates velocity data of the sPIV unit, the differential pressure probes as well as the true airspeed that was acquired by the onboard airspeed indicator of the aircraft itself. The presented time series presents a drastic example of the dependence of sPIV data quality on the particle image densities. The depicted cloud droplet concentration is based on the averaged particle counts within $64 \mathrm{px} \times 64 \mathrm{px}$ windows. The number of cloud droplets within these windows drops down to a few tracer particles per interrogation window at times and can be as high as 70 particles per interrogation window.

The laser light sheet and the Pitot tube were positioned $72 \mathrm{~mm}$ away from the wall at roughly $y^{+}=10,496$. The viscous units of the Pitot probe and the smallest interrogation window size of this measuring cycle (C141 in Table 1) are identical. The data of all measurement systems was averaged over $3 \mathrm{~s}$. The same sequence of processing steps was employed to the sPIV images of the time series. The sole difference was the modification of the final interrogation window size. The most drastic discrepancy between the observations occurred when the particle density dropped below a critical value and the cross-correlation algorithm failed to process the particle images. Adequate particle densities resulted in a good agreement concerning the SPIV velocities. The deviations between the sPIV results and the Pitot probe velocities seemed quite constant throughout the entire time series. This observation points to a systematic error and will be discussed in Sect. 4.5.
Table 1 Resolution of the measurement systems in viscous units

\begin{tabular}{|c|c|c|c|c|c|c|c|c|}
\hline \multirow[t]{2}{*}{ Case } & \multirow[t]{2}{*}{$M_{\mathrm{f}}[\mathrm{px} / \mathrm{mm}]$} & \multirow[t]{2}{*}{$u_{\tau}[\mathrm{m} / \mathrm{s}]$} & \multicolumn{2}{|c|}{ Pressure Probes } & \multicolumn{4}{|l|}{ sPIV } \\
\hline & & & $d_{\mathrm{blp}}^{+}$ & $d_{\mathrm{pp}}^{+}$ & $\mathrm{i}^{+}: 48 \mathrm{px}$ & $\mathrm{i}^{+}: 64 \mathrm{px}$ & $\mathrm{i}^{+}: 128 \mathrm{px}$ & $\mathrm{b}_{\text {sPIV }}^{+}$ \\
\hline 152 & 19 & 2.053 & $145 \times 290$ & 362 & 306 & 407 & 814 & 242 \\
\hline 150 & 19 & 2.510 & $177 \times 354$ & 443 & 374 & 498 & 995 & 295 \\
\hline 126 & 16 & 2.459 & $184 \times 369$ & 461 & 461 & 615 & 1230 & 307 \\
\hline 008 & 16 & 2.566 & $171 \times 342$ & 428 & 428 & 570 & 1140 & 285 \\
\hline (141) & 16 & 2.605 & $175 \times 350$ & 437 & 437 & 583 & 875 & 292 \\
\hline 143 & 16 & 3.373 & $225 \times 450$ & 562 & 562 & 750 & 1499 & 375 \\
\hline 142 & 16 & 3.405 & $227 \times 454$ & 568 & 568 & 757 & 1513 & 378 \\
\hline 154 & 19 & 3.467 & $245 \times 490$ & 612 & 516 & 687 & 1375 & 408 \\
\hline
\end{tabular}

The interrogation window size is listed as $\mathrm{i}^{+}$. The sPIV interrogation windows are squared, hence only one side length is listed. The kinematic viscosity $v$ was $1.6-1.8 \cdot 10^{-5} \mathrm{~m}^{2} / \mathrm{s}$. Case $\mathrm{C} 141$ will not be used for the boundary layer analysis due to its inconsistent particle density. The two dimensions of the boundary layer probe $d_{\mathrm{blp}}^{+}$refer to the different diameters of the flattened opening 
Fig. 4 Time series of C141 that conflates velocity measurements of three subsystems (differential pressure probes, sPIV, aircraft system). The sPIV results were derived with varying interrogation windows sizes. The droplet density estimation is based on the average number of particle images within interrogation windows of size $64 \mathrm{px} \times 64 \mathrm{px}$

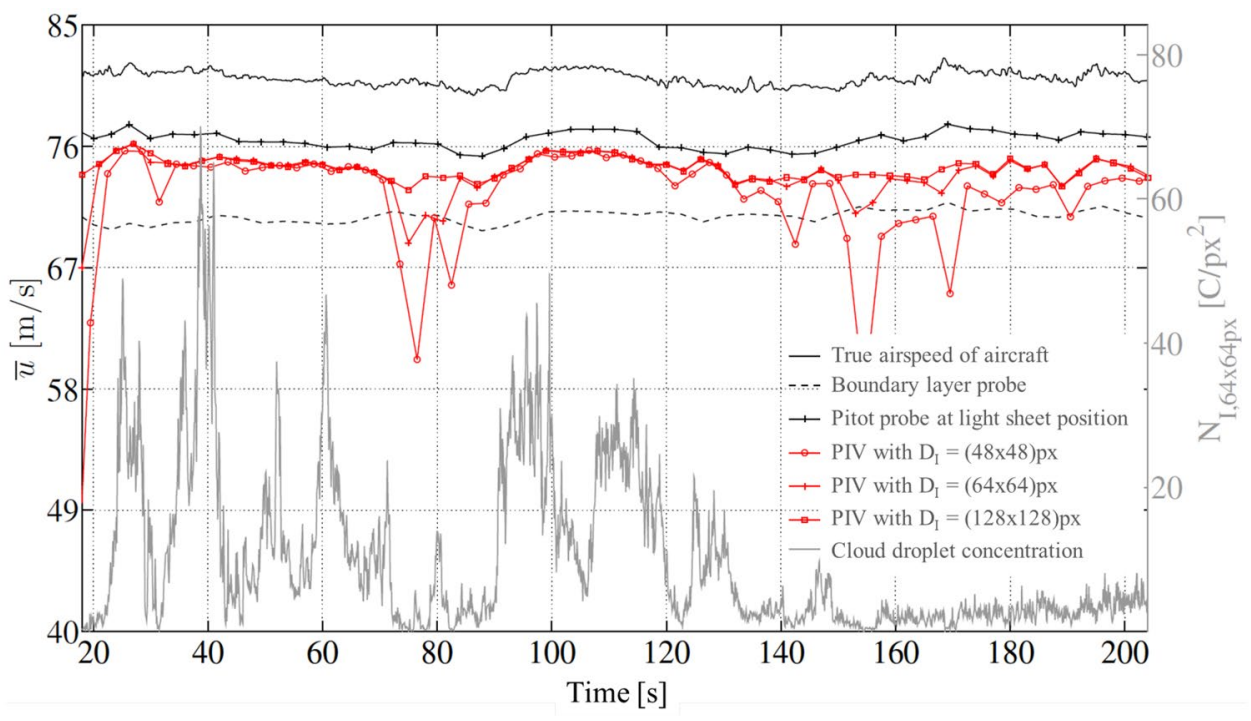

\subsection{Impact of the droplet dynamics on SPIV results}

The assessment of the hydrometeors that served as tracer particles for sPIV was based on ILIDS observations. The evaluation of the ILIDS data revealed a dependence of the concentration and droplet size distribution on the encountered cloud genera. Shallow stratified clouds exhibited lower levels of particle concentrations whereas towering Cumulus clouds showed significantly higher particle density that ranged between a few hundreds to more than 1300 particles $/ \mathrm{cm}^{3}$. The density distributions of the droplet diameters in stratified clouds were characterised by a narrow size spectra and partially bimodal shapes. The distribution in Cumulus clouds appeared broader with slightly higher average diameters. The mean particle diameters of the observed cloud genera varied between $11 \mu \mathrm{m}$ and $18 \mu \mathrm{m}$ with standard deviations of up to $0.5 \mu \mathrm{m}$.

The response time and velocity fidelity of tracer particles in turbulent flow was estimated based on a simplified form of the Maxey-Riley equation (Mei 1996). The derived equation incorporates the Stokes drag in quasi-steady form; body forces (gravity minus buoyancy); as well as a force that accounts for the added mass. The resulting second-order ordinary differential equation was solved with the adaptive Runge-Kutta-Fehlberg method. This approach was applied to an instantaneous excerpt of differential pressure probe data. Different droplet diameters were selected as input parameters to contrast the impact of larger particles on the sPIV measurement. Figure 5 highlights the differences in response times and velocities between the streamwise velocity component of the fluid $u_{\mathrm{f}}$ and the cloud droplets $u_{\mathrm{p}, W}$. The full spectra of the flow might not be replicated to its fullest extend since both differential pressure systems experienced pressure attenuations. Hence, the accelerations that are imposed on the particles might be less severe compared to actual wall bounded turbulence. Droplet diameters of $8 \mu \mathrm{m}$,

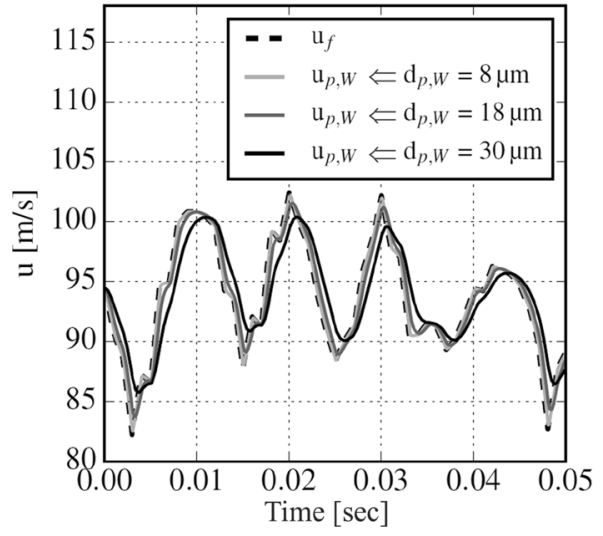

Fig. 5 Velocity differences in streamwise direction between the fluid $u_{\mathrm{f}}$ and cloud droplets $u_{\mathrm{p}, W}$ of different diameters

$18 \mu \mathrm{m}$ and $30 \mu \mathrm{m}$ were selected for the assessment of the velocity fidelity. The slip velocities of the $8 \mu \mathrm{m}$ and $18 \mu \mathrm{m}$ are less than $2 \mathrm{~m} / \mathrm{s}$. Velocity differences of $3 \%$ are likely in clouds where particles with diameters of more than $30 \mu \mathrm{m}$ are dominant. This deviation is less critical for averaged results where the contributions of isolated accelerations in the flow diminish. Furthermore, the mean diameter of most encountered cloud genera were significant lower than $30 \mu \mathrm{m}$ and the impact of the droplet dynamics on the SPIV measurement will hence succumb to the quality loss of the data due to low droplet concentration (see Section 4.3).

\subsection{Differences in measured velocities between sPIV and pressure probes}

An obvious question is: how well does the obtained velocity information of the sPIV corroborate the differential pressure probes data? The average velocity difference 
between the two systems was roughly $2-3 \mathrm{~m} / \mathrm{s}$ or $3-4 \%$. The reasons for these discrepancies in accuracy are manifold. As aforementioned combining single-point velocity data with sPIV can pose the risk of applying insufficient averaging intervals in the temporal or spatial domain. This will manipulate the dynamics and influence the uncertainty. The disadvantage of using hydrometeors as a a seeding source was the highly variable availability of particles within the field of view. Numerous measuring cycles exhibited a poor correlation due to missing particles within the field of view; furthermore, disparity effects and velocity gradients impaired the sPIV outcome. Raffel et al. (2004) introduced a simple equation to estimate the accuracy of a PIV setup. Following this approach leads to a PIV accuracy of around $1-2 \%$ if the seeding concentration is above a minimum threshold of 10 particles per interrogation window. The most critical data correction measures, with respect to the differential pressure probes, were the offset voltage modification by incorporating the aircraft total pressure information and the utilisation of the Clauser chart method for the derivation of the boundary layer parameters. Hence, the inaccuracy of the pressure probe system, due to mainly systematic errors, can be expected to be at least $\pm 5 \%$.

\section{Results}

\subsection{Mean flow}

Seven measuring cycles are selected to represent the flight test data set. As aforementioned, the variation in airspeed during the scanning transit of the stem through the boundary layer is the most stringent selection criteria. However, airspeed variations are inevitable in flight testing, especially when flying through clouds and at lower airspeeds. Figure $6 \mathrm{a}$ depicts the mean velocity profile of a selection of cases as a function of the measurement position. The velocity information of each data point were averaged over three seconds. The dashed lines present the prevailing airspeeds during the scanning of the boundary layers. As expected the cases no. C152 and C008, which were acquired at lower airspeeds, exhibit distortions of the profiles caused by airspeed variations of up to $3.0 \mathrm{~m} / \mathrm{s}$ while the stem was driving through the boundary layer.

This deviation from a clean profile influences the derivation of boundary layer parameters, in particular the integral measures as well as the boundary layer thickness $\delta_{99}$ as listed in Table 2, which was taken as the value $y$ at $99 \%$ of the free-stream velocity $u_{\infty}$. The chosen measuring cycles cover a Reynolds number $\mathrm{Re}_{\theta}$ regime (based on momentum
Fig. 6 a Mean streamwise velocity profiles of five selected cases at varying flight conditions (see details in Table 2).

The dashed lines indicate the true airspeed during each boundary layer scan. bThe same mean velocity profiles normalised by the viscous scales. Friction velocities are derived by means of the Clauser chart method. The dashed line presents the logarithmic law with $\kappa=0.384$ and $\mathrm{B}=4.171$

Table 2 Experimental parameters of selected measuring cycles
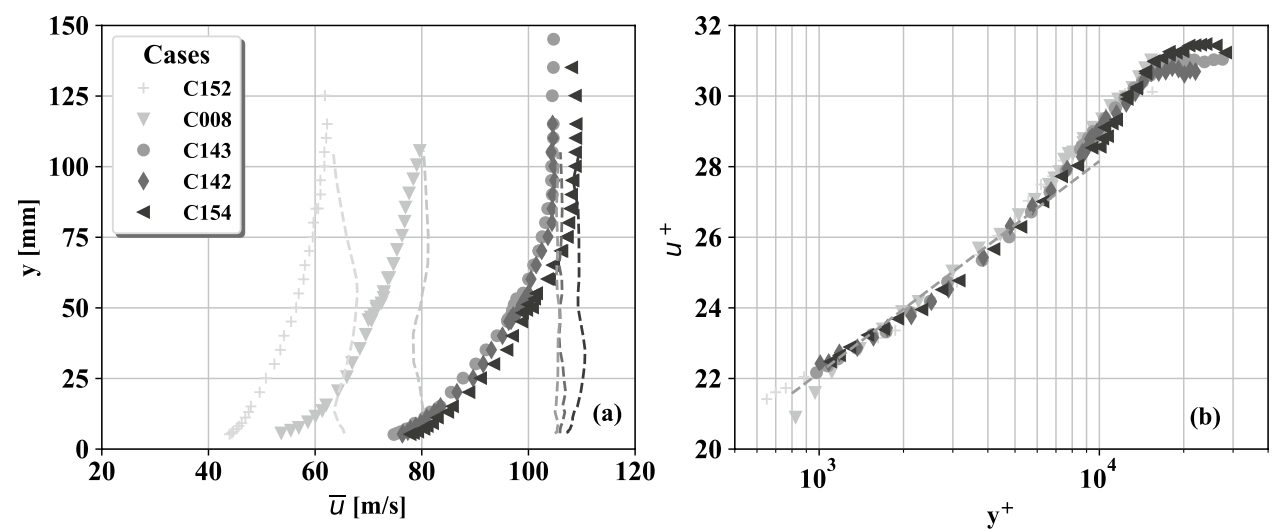

\begin{tabular}{lllllllllll}
\hline $\begin{array}{l}\text { Case } \\
{[-]}\end{array}$ & $\begin{array}{l}\text { Flaps } \\
{\left[{ }^{\circ}\right]}\end{array}$ & $\begin{array}{l}\mathrm{u}_{\infty} \\
{[\mathrm{m} / \mathrm{s}]}\end{array}$ & $\begin{array}{l}\delta_{99} \\
{[\mathrm{~mm}]}\end{array}$ & $\begin{array}{l}\mathrm{Re}_{x} \\
{\left[\cdot 10^{6}\right]}\end{array}$ & $\begin{array}{l}\mathrm{Re}_{\theta} \\
{[-]}\end{array}$ & $\begin{array}{l}\mathrm{u}_{\tau} \\
{[\mathrm{m} / \mathrm{s}]}\end{array}$ & $\begin{array}{l}\mathrm{Tu} \\
{[\%]}\end{array}$ & $\begin{array}{l}\mathrm{Re}_{\tau} \\
{[-]}\end{array}$ & $\begin{array}{l}\text { Altitude } \\
{[\mathrm{m}]}\end{array}$ & $\begin{array}{l}\mathrm{Ma} \\
{[-]}\end{array}$ \\
\hline 152 & 20 & 60.8 & 85.2 & 32.98 & 23,794 & 2.053 & 1.8 & 10,525 & 1470 & 0.2 \\
150 & 0 & 75.6 & 111.0 & 39.00 & 44,314 & 2.510 & 1.0 & 16,008 & 1480 & 0.23 \\
126 & 0 & 76.1 & 110.3 & 40.42 & - & 2.459 & 1.2 & 15,583 & 1110 & 0.23 \\
008 & 0 & 79.3 & 103.2 & 38.44 & 37,100 & 2.566 & 3.8 & 14,928 & 2369 & 0.25 \\
143 & 0 & 104.7 & 85.2 & 49.51 & 41,336 & 3.373 & 1.0 & 16,142 & 2370 & 0.32 \\
142 & 0 & 105.2 & 80.2 & 49.94 & 40,384 & 3.405 & 1.1 & 15,337 & 2370 & 0.32 \\
154 & 0 & 108.8 & 85.1 & 55.12 & 49,403 & 3.467 & 1.2 & 17,764 & 1468 & 0.33 \\
\hline
\end{tabular}


thickness $\theta$ ) from 23,794 to 49,403 with true airspeeds $\mathbf{u}_{T A S}$ between $66 \mathrm{~m} / \mathrm{s}$ to $109 \mathrm{~m} / \mathrm{s}$ that correspond to Mach numbers ranging from $\mathrm{Ma}=0.2$ to 0.33 . The maximum Reynolds number $\operatorname{Re}_{x}$ achieved was $5.51 \cdot 10^{7}$ and the friction velocity $\mathrm{u}_{\tau}$ varied between 2.053 and 3.467 resulting in a Reynolds number range based on friction velocities between 10,525 and 17,764 .

The Clauser chart method (Clauser 1956) is used to determine the friction velocity $\mathrm{u}_{\tau}$ and hence to further expand the analysis of the velocity profiles and the turbulence intensities. The given data set deviates to a certain extent from the logarithmic law of the velocity profile; increased friction on the outer fuselage surface is not incorporated in this analysis; and the high Reynolds number cases require a judicious selection of the von Kármán constant $\kappa$ as well as the constant B. The latter requirement is addressed by following the argumentation of Buschmann and Gad-El-Hak (2003) that refers to high Reynolds number data of Österlund et al. (2000). For the purpose of this study, the two constants are hence chosen to be $\kappa=0.384$ and $B=4.171$. The resulting boundary layer profiles plotted in terms of inner variables $\mathrm{u}^{+}=\bar{u} / \mathrm{u}_{\tau}$ and $\mathrm{y}^{+}=y u_{\tau} / v$ are depicted in Fig. $6 \mathrm{~b}$. The minimum distance of the pressure probes from the wall was $y^{+}=650$. The onset of the departure from the logarithmic law in the outer region is evident and presents itself with a wake-like shape with upward and downward turning tendencies. The derived friction velocity $\mathrm{u}_{\tau}=\mathrm{u}_{e} \sqrt{c_{f} / 2}$ of each plotted case are listed in Table 2. The imperfections of the data result in an inaccuracy of the estimation of the friction coefficient $\mathrm{c}_{f}$ of $\pm 5 \%$.

A scrutiny of the wake region of the boundary layer in Fig. $6 \mathrm{~b}$ reveals shapes partially deviating from the law of the wake as introduced by Coles (1956). Figure 7a replots the velocity profiles of Fig. 6a,b. This figure highlights the deviation of the profiles due to the wake by subtracting the logarithmic law $u_{\log }^{+}$from the dimensionless velocity $\mathrm{u}^{+}$. The negative defect closest to the logarithmic law region can be linked to a curved velocity profile in the overlap region (Fig. 6b). This retardation of the boundary layer flow is no longer present in the defect layer beyond $\eta=y / \delta=0.2$, where the velocity deficit translates to a positive departure from the logarithmic law. The cause for
Fig. 7 a The mean velocity profiles replotted with outer law variables highlighting the deviation of the data from the logarithmic law. aStreamwise turbulence fluctuations. The enlarged red marker is a sPIV result of the corresponding case. The turbulence intensity varied between $1.0 \%$ for $\mathrm{C} 150$ and $1.2 \%$ for $\mathrm{C} 126$ (please refer to Table 2)

Fig. 8 Smoothed energy spectra of the instantaneous pressure data as acquired from the boundary layer probe (a) and the Pitot probe (b). The selected measuring cycle is C143. Each figure depicts three different wall distances to contrast the changes in the amplitudes. The vertical dashed line marks the frequency of rotation of the 5-bladed propeller whereas the solid vertical line highlights the estimated Helmholtz resonance frequency
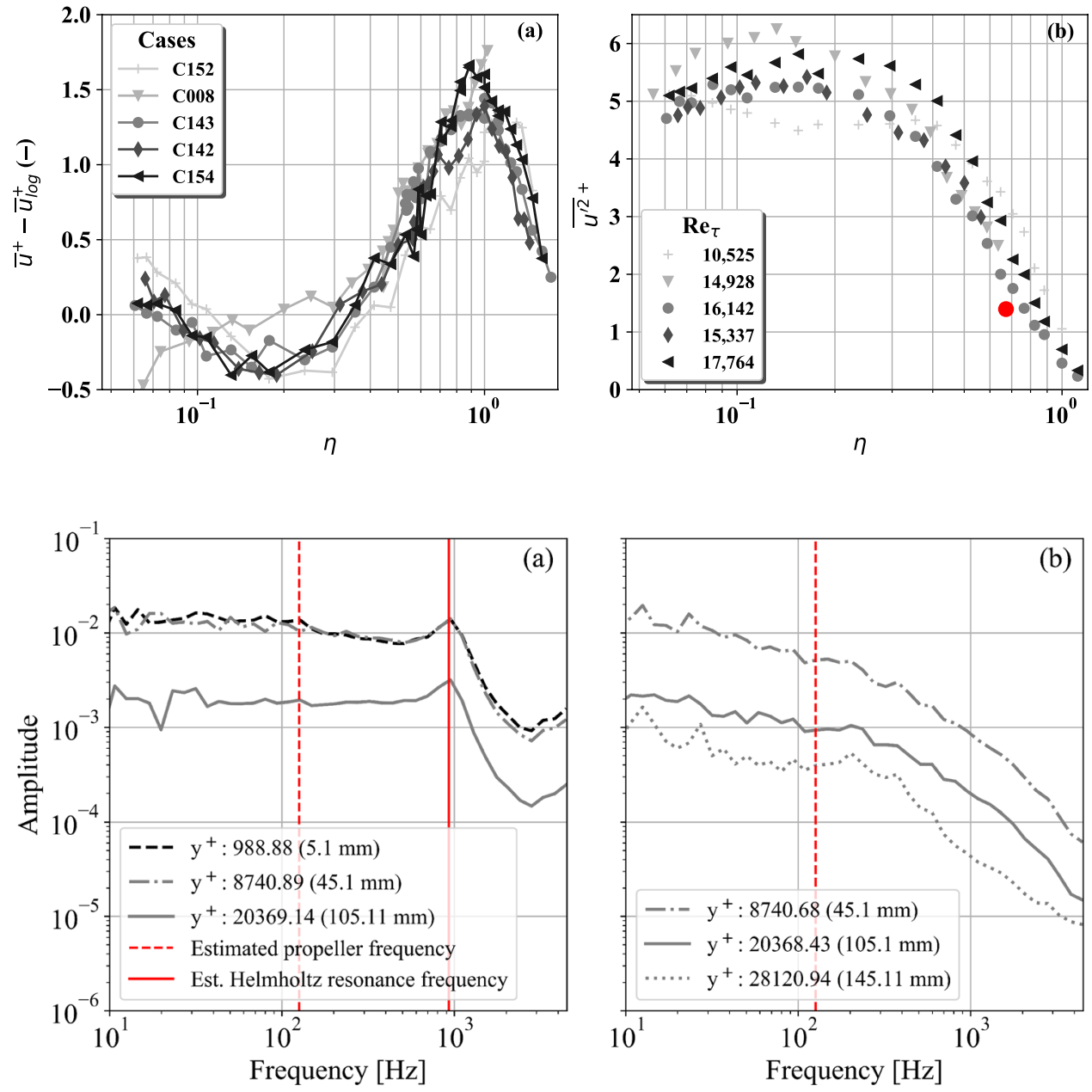
this anomaly is not clear and it might point to either a systematic error in the measurement, changes in pressure gradients or an increased level of atmospheric turbulence while flying through clouds.

The turbulence intensity level $\mathrm{Tu}$ of the atmosphere, as listed in Table 2, is calculated based on the velocity fluctuations and the mean velocities at the outermost position of the inner Pitot tube, the boundary layer probe. The differential pressure sensor of this inner boundary layer probe was less affected by the attenuation of the pressure information that is caused by pneumatic tubing. The disadvantage is that the inner probe was not always pushed beyond $\delta_{99}$ and hence Tu was in some cases influenced by the inherent turbulence intensities of the wall-bounded flow. The resulting turbulence levels are in some cases slightly increased and the turbulence mechanisms of various dimensions have, therefore, a verifiable impact on the shape of the mean velocity profile. Thole and Bogard (1996) linked an increase of the free-stream turbulence to a diminishing wake strength, but so does an increasing pressure gradient. The scarce amount of data does not support the identification of an obvious causality that would explain the systematics of the excess velocities of the mean velocity profiles. The influence of the propeller can be eliminated as potential source since the energy spectra are not presenting any dominant frequencies that correspond to the propeller speeds (see Fig. 8). Based on the information at hand (Dornier Luftfahrt GmbH 1991) an additional energy contribution that is linked to the rotational frequency of the 5-bladed propeller would appear around $126 \mathrm{~Hz}$. No distinct peak at that frequency can be found in the unprocessed as well as the smoothed spectra.

\subsection{Turbulent Intensities}

Solely the inner boundary layer probe is used for the analysis of broadband turbulent intensities since the sensor of this installation was placed as close as possible to the mouth of the probe. The obtained streamwise Reynolds stresses ${\overline{u^{\prime 2}}}^{+}=\overline{u^{\prime 2}} / u_{\tau}^{2}$ are shown in Fig. 7b. A distinct peak roughly between $\eta=0.1$ and $\eta=0.2$ indicates a dependency of this secondary outer peak location and its intensity on the Reynolds number $\operatorname{Re}_{\tau}$ or on the viscous scales respectively as discussed in Sect. 4.3. The magnitudes of streamwise variances as well as the inflection points appear closer to the wake region with viscous units. This increase of the outer ${\overline{u^{\prime 2}}}^{+}$-peak with growing Reynolds number is confirmed by many studies, although some of those studies (DeGraaff and Eaton 2000; Hutchins et al. 2009; Marusic et al. 2010) demonstrated the position of the peak can be affected by poor spatial resolution that does not resolve all the near-wall velocity fluctuations.

A similar effect can occur when using pitot tubes with a flattened opening: eddies smaller than the probe opening may be damped by this spatial filter and hence their contribution to the energy budget is distorted. The turbulence intensities exhibit a strong tendency to collapse at the probe positions closest and farthest from the wall. The spatial and temporal attenuation due to the measurement resolution of the sPIV and the differential pressure units prevents a clear distinction of the potential causality in the outer hump. It is however likely that the inherently coarse resolution in viscous units are defining the outer peak characteristics.

One of the presented cases provides instantaneous sPIV data with an acceptable seeding density as well as low levels of atmospheric turbulence. The variance of the case C143 is retrieved by averaging the streamwise velocity component over 100 images with an interrogation window size of $48 \mathrm{px} \times 48$ pixel (measurement plane at $\mathrm{y}_{P I V, \text { max }}=72 \mathrm{~mm}, 75 \%$ overlap, multigrid interrogation, Whittaker reconstruction, disparity correction) and by scaling with the respective friction velocity as listed in Table 2 . The deviation between the sPIV observations and the Pitot tube data is caused by the spatial attenuation of the SPIV unit. The resolution in viscous units are twice as large compared to the boundary layer probe (see Table 1).

In general, experimental data that does not only comprise velocity fluctuations at high Reynolds numbers in $u^{\prime}$ (streamwise) but also in $v^{\prime}$ (normal) and $w^{\prime}$ (lateral) directions is rather sparse. The on-board sPIV system with its low temporal but high spatial extensions compared to single-point measurements catched a glimpse of these velocity components (Politz 2017). A sample result is depicted in Fig. 9 and it highlights the increasing turbulent intensities in streamwise and lateral directions closer to the wall.

Both observations were recorded at similar true airspeeds of around $76 \mathrm{~m} / \mathrm{s}$ as well as at comparable flight levels. The light sheet position was moved from $\mathrm{y}_{P I V, C 150}=41.5 \mathrm{~mm}$ further outwards to $\mathrm{y}_{P I V, C 126}=72 \mathrm{~mm}$ and hence enabled the investigation of turbulence within the outer layer and the wake region. The Reynolds numbers vary slightly between $\operatorname{Re}_{\tau, 150}=16,008$ and $\operatorname{Re}_{\tau, 126}=15,583$. In this example, the mean flow velocities in streamwise directions deviate by $8.2 \mathrm{~m} / \mathrm{s}$. It is noticeable that the turbulent intensities are significantly higher closer to the wall. Complementary statistical parameters are based on sample sizes that comprise more than 100 images.

\section{Conclusions}

The feasibility of an airborne research platform for in-situ turbulent research involving a full-scale aircraft is scrutinised with this study. The present flight test experiment provides observations of all three velocity components acquired within a turbulent boundary layer at high Reynolds number; thus contributing to an under-explored field 

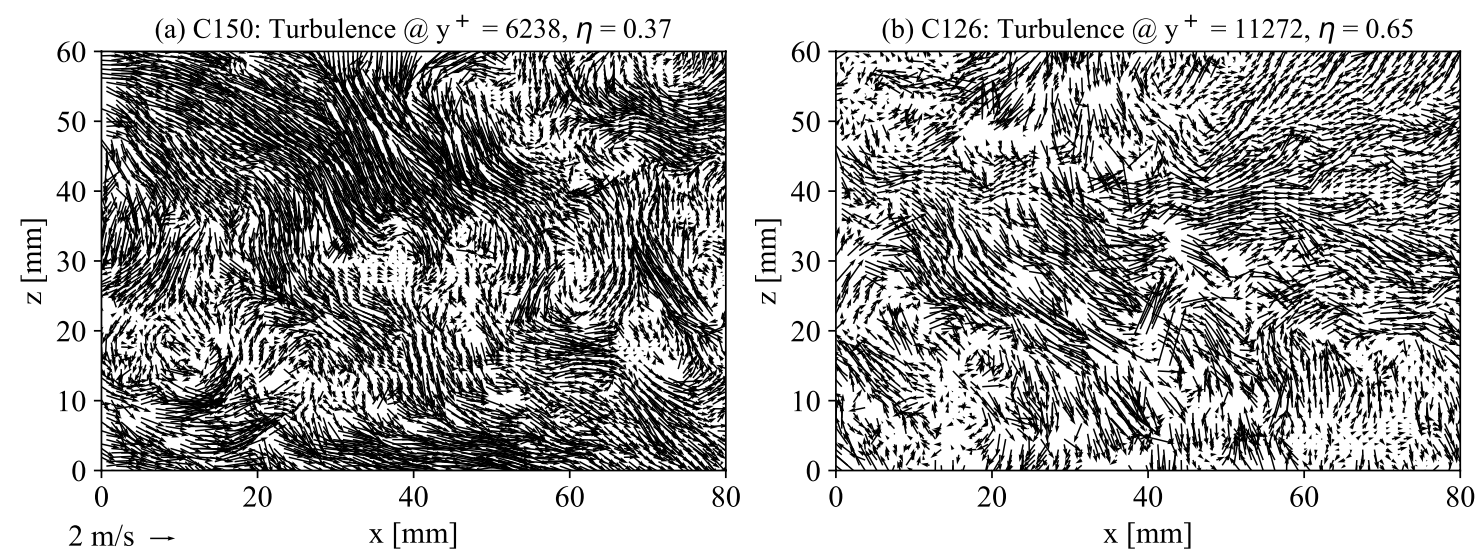

Fig. 9 Instantaneous turbulence intensities $u^{\prime} / u_{\tau}$ and $w^{\prime} / u_{\tau}$ at different distances away from the wall (every second vector is plotted)

of turbulence research. The boundary conditions place the turbulent boundary layer in an authentic full-scale scenario with high relevance to various engineering sciences and applications. This data set covers the outer layer and the wake region that partially resembles a canonical equilibrium boundary layer. Deviations from the canonical equilibrium boundary layer in the wake region could be attributed to measurement errors, undetected pressure gradients or increased levels of atmospheric turbulence. An additional array of pressure sensors could have provided complementary information on the prevailing pressure gradients to support the findings. The quality of the data was mainly dictated by atmospheric conditions and the stability of the aircraft. Future attempts to investigate a boundary layer with a similar setup could significantly improve the outcome by implementing artificial seeding generators with particle outlets upstream of the measurement area. This approach would supersede the necessity for flights through clouds which exhibit in general a higher turbulence level. In summary, this proof-of-concept study illustrated the expedient capabilities of a small-sized aircraft with its limited flight envelope in combination with a comparably simple setup for turbulent flow research and hence introduces a new type of facility with access to a recondite flow regime.

Acknowledgements The herewith presented work was coordinated and executed by the DLR department Experimental Methods. It was funded by the European Commission within the 7th Framework Programme as part of the project Advanced In-Flight Measurement Techniques 2 $\left(\mathrm{AIM}^{2}\right)$. The author would gratefully acknowledge the support provided by the DLR Institute of Aerodynamics and Flow Technology, in particular the DLR department Experimental Methods and namely Dr Reinhard Geisler, Dr Andreas Schröder, Dr Lars Koop, Carsten Fuchs, Janos Agocs, Tobias Kleindienst and Florian Philipp. The author is also thankful for the support by Andreas Grüttemann of the DLR Institute of Flight Guidance, Dr Klaus Ehrenfried (DLR, Ground Vehicles) and the DLR facility Flight Experiments. The author would also like to acknowledge the excellent collaboration between DLR and Cranfield University and in particular the support provided by Prof. Nicholas Lawson. The author would like to thank the anonymous referees for their valuable comments.

Funding Open Access funding enabled and organized by Projekt DEAL.

\section{Compliance with ethical standards}

Conflict of interest The authors declare that they have no conflict of interest.

Open Access This article is licensed under a Creative Commons Attribution 4.0 International License, which permits use, sharing, adaptation, distribution and reproduction in any medium or format, as long as you give appropriate credit to the original author(s) and the source, provide a link to the Creative Commons licence, and indicate if changes were made. The images or other third party material in this article are included in the article's Creative Commons licence, unless indicated otherwise in a credit line to the material. If material is not included in the article's Creative Commons licence and your intended use is not permitted by statutory regulation or exceeds the permitted use, you will need to obtain permission directly from the copyright holder. To view a copy of this licence, visit http://creativecommons.org/licenses/by/4.0/.

\section{References}

Andreas EL, Claffey KJ, Jordan RE, Fairall CW, Guest PS, Persson POG, Grachev AA (2006) Evaluations of the von Kármán constant in the atmospheric surface layer. J Fluid Mech 559:117-149

Bailey SCC, Hultmark M, Monty JP, Alfredsson PH, Chong MS, Duncan RD, Fransson JHM, Hutchins N, Marusic I, McKeon BJ, Nagib HM, Örlü R, Segalini A, Smits AJ, Vinuesa R (2013) Obtaining accurate mean velocity measurements in high Reynolds number turbulent boundary layers using Pitot tubes. J Fluid Mech 715:642-670

Buschmann MH, Gad-El-Hak M (2003) Debate concerning the meanvelocity profile of a turbulent boundary layer. AIAA J 41:565-572 
Clauser FH (1956) The turbulent boundary layer. Adv Appl Mech $4: 1-51$

Coles D (1956) The law of the wake in the turbulent boundary layer. J Fluid Mech 1:191-226

DeGraaff DB, Eaton JK (2000) Reynolds-number scaling of the flatplate turbulent boundary layer. J Fluid Mech 422:319-346

Dornier Luftfahrt GmbH (1991) Dornier 228-101 - Pilot's Operating Handbook Including the LBA Approved Airplane Flight Manual, Revision No. 3, Serial No. 7083

Dunker C, Roloff C, Grassmann A (2016) Interferometric laser imaging for in-flight cloud droplet sizing. Meas Sci Technol 27:1-10

Dunker C, Geisler R (2018) Full-scale in-flight flow investigation of a high-lift vortex system by means of particle image velocimetry. In: Dillmann A et al (eds) New results in numerical and experimental fluid mechanics XI. Notes on numerical fluid mechanics and multidisciplinary design, vol 136 . Springer, Berlin

Gyorgyfalvy D (1965) Effect of pressurization on airplane fuselage drag. J Aircraft 2:531-537

Heisel M, Dasari T, Liu Y, Hong J, Coletti F, Guala M (2018) The spatial structure of the logarithmic region in very-high-Reynolds-number rough wall turbulent boundary layers. J Fluid Mech 857:704-747

Hommema SE, Adrian RJ (2003) Packet structure of surface eddies in the atmospheric boundary layer. Boundary-Layer Meteorol. 106: $147-170$

Hutchins N, Nickels TB, Marusic I, Chong MS (2009) Hot-wire spatial resolution issues in wall-bounded turbulence. J Fluid Mech 635:103-136

Hutchins N, Chauhan K, Marusic I, Monty J, Klewicki J (2012) Towards reconciling the large-scale structure of turbulent boundary layers in the atmosphere and laboratory. Boundary-Layer Meteorol 145:273-306

Lui HY, Bo TL, Liang YR (2017) The variation of large-scale structure inclination angles in high Reynolds number atmospheric surface layers. Phys Fluids 29:035104

Marusic I, McKeon BJ, Monkewitz PA, Nagib HM, Smits AJ, Sreenivasan KR (2010) Wall-bounded turbulent flows at high Reynolds numbers: recent advances and key issues. Phys Fluids 22:065103

McKeon BJ, Li J, Jiang W, Morrison JF, Smits AJ (2003) Pitot probe corrections in fully developed turbulent pipe flow. Meas Sci Technol 14:1449-1458

Mei R (1996) Velocity fidelity of flow tracer particles. Exp Fluids 22:1-13

Metzger M, McKeon BJ, Holmes H (2007) The near-neutral atmospheric surface layer: turbulence and non-stationarity. Phil Trans R Soc Lond A 365:859-876
Örlü R, Fiorini T, Segalini A, Bellani G, Talamelli A, Alfredsson PH (2017) Reynolds stress scaling in pipe flow turbulence-first results from CICLoPE. Phil Trans R Soc A 375:1-14

Österlund JM, Johansson AV, Nagib HM, Hites MH (2000) A note on the overlap region in turbulent boundary layers. Phys Fluids $12: 1-4$

Politz C, Lawson NJ, Konrath R, Agocs J, Schröder A (2013) Development of particle image velocimetry for in-flight flow measurement. In: Boden F, Lawson NJ, Jentink H, Kompenhans J (eds) Advanced in-flight measurement techniques research topics in aerospace. Springer, Berlin, pp 269-289

Politz C (2017) Anwendung des optischen Geschwindigkeitsmessverfahrens Particle Image Velocimetry im Freiflug zur Charakterisierung einer Grenzschichtströmung Ph.D. thesis, Technischen Universität Berlin

Raffel M, Richard H, Ehrenfried K, Van der Wall B, Burley C, Beaumier P, McAlister K, Pengel K (2004) Recording and evaluation methods of PIV investigations on a helicopter rotor model. Exp Fluids 36:146-156

Rudnik R, Schwetzler D (2016) High lift INflight VAlidation (HINVA) - Overview about the 2nd Flight Test Campaign, AIAA SciTech, San Diego, 4-8 January

Thole KA, Bogard DG (1996) High feestream turbulence effects on turbulent boundary layers. ASME J Fluids Eng 118(2):276-284

Toloui M, Riley S, Hong J, Howard K, Chamorro LP, Guala M, Tucker J (2014) Measurement of atmospheric boundary layer based on super-large-scale particle images velocimetry using natural snowfall. Exp Fluids 55:1737

Utama IKAP, Nugroho B, Baidya R, Nurrohman MN, Yusim AK, Hakim ML, Prasetyo FA, Yusuf M, Suastika IK, Ganapathisubramani B, Monty JP, Hutchins N (2018) Skin-friction drag measurement over a recently cleaned and painted ship hull under steady cruising via in-situ laser-based measurement coupled with empirical estimation, RINA International Conference: Full Scale Ship Performance, London, 24-25 October

Zagarola M, Smits AJ (1998) Mean-flow scaling of turbulent pipe flow. J Fluid Mech 373:33-79

Wang G, Zheng X (2016) Very large scale motions in the atmospheric surface layer: a field investigation. J Fluid Mech 802:464-489

Publisher's Note Springer Nature remains neutral with regard to jurisdictional claims in published maps and institutional affiliations. 